\title{
Erratum to: Pituitary tumors and pregnancy: the interplay between a pathologic condition and a physiologic status
}

\author{
Rosario Pivonello - Maria Cristina De Martino - Renata S. Auriemma • \\ Carlo Alviggi - Ludovica F. S. Grasso • Alessia Cozzolino • Monica De Leo • \\ Giuseppe De Placido • Annamaria Colao · Gaetano Lombardi
}

Published online: 1 May 2014

(C) Italian Society of Endocrinology (SIE) 2014

\section{Erratum to: J Endocrinol Invest (2014) 37:99-112 DOI 10.1007/s40618-013-0019-8}

In the published original article, in Table 6, column "References" and column "Child", some information are not published correctly. The correct table is given here.

In the title of Table 4 the right sentence should be: "adapted from Ref. 51".
In the title of Table 5 the right sentence should be: "adapted from Ref. 54 and 55".

In the notes of Table 4 and 5 the right sentence should be: "Data on worldwide general population regarding spontaneous abortion have been extrapolated from Sedgh et al. [94]".

The online version of the original article can be found under doi:10.1007/s40618-013-0019-8.

R. Pivonello - M. C. De Martino - R. S. Auriemma .

L. F. S. Grasso - A. Cozzolino - M. De Leo - A. Colao ·

G. Lombardi

Section of Endocrinology, Department of Clinical Medicine and

Surgery, "Federico II" University, Naples, Italy

R. Pivonello $(\bowtie)$

Department of Molecular and Clinical Endocrinology and Oncology, "Federico II" University, Via S. Pansini 5,

80131 Naples, Italy

e-mail: rosario.pivonello@unina.it

C. Alviggi · G. De Placido

Section of Gynaecology, Department of Neurosciences,

Reproductive and Odontostomatologic Sciences, "Federico II"

University, Naples, Italy 
Table 6 Acromegaly and pregnancy: overview of literature

\begin{tabular}{|c|c|c|c|c|c|c|c|c|}
\hline \multirow[t]{2}{*}{ Author, year } & \multirow[t]{2}{*}{ References } & \multirow[t]{2}{*}{ Pregnancy $\mathrm{Nr}$} & \multicolumn{2}{|l|}{ On therapy } & \multicolumn{2}{|c|}{ Treatment ongoing } & \multicolumn{2}{|l|}{ Complications } \\
\hline & & & SA & DA & SA & DA & Mother & Child \\
\hline Colao, 1997 & [74] & $\begin{array}{l}10 \text { (1 } \\
\text { spontaneous } \\
\text { abortion; } 1 \\
\text { pregnancy still } \\
\text { ongoing at } \\
\text { data analysis) }\end{array}$ & 2 & 0 & 1 & 0 & 0 & 0 \\
\hline Cozzi R, 2006 & {$[37]$} & 7 & 2 & 0 & 0 & 0 & 0 & 0 \\
\hline Atmaca, 2006 & [73] & $\begin{array}{c}7 \text { (2 therapeutic } \\
\text { abortions) }\end{array}$ & $\begin{array}{l}1 \text { (1 } \\
\text { therapeutic } \\
\text { abortion) }\end{array}$ & 3 & 0 & 0 & $\begin{array}{l}3 \text { (pituitary apoplexy in one } \\
\text { case; gestational diabetes } \\
\text { mellitus in } 2 \text { cases) }\end{array}$ & 0 \\
\hline Caron, 2010 & [81] & 59 & 14 & 25 & 14 & 25 & $\begin{array}{l}12 \text { (gestational diabetes in } 4 \\
\text { cases; gravid hypertension } \\
\text { in } 5 \text { cases; preeclampsia in } \\
3 \text { cases, } 2 \text { of which in the } \\
\text { same patient) }\end{array}$ & $\begin{array}{l}12 \text { (preterm birth in } \\
4 \text { cases; } \\
\text { macrosomia in } 2 \\
\text { cases and low } \\
\text { birth weight in } 6 \\
\text { cases) }\end{array}$ \\
\hline Cheng, 2012 & {$[82]$} & 13 & 11 & 6 & 3 & 0 & 0 & $\begin{array}{l}1 \text { (small for } \\
\text { gestational age) }\end{array}$ \\
\hline Total & & 96 & $30(31.3 \%)$ & $34(35.4 \%)$ & $18(18.7 \%)$ & $25(26 \%)$ & $15(15.6 \%)$ & $13(13.5 \%)$ \\
\hline
\end{tabular}

